Die Statistik der Parahotellerie erwies sich im Laufe der sechziger Jahre als eine Notwendigkeit, um die atemraubende Nachfrageentwicklung auf diesem Sektor zahlenmäßig verfolgen zu können. Das Eidgenössische Departement des Innern verlangte daher im Jahre 1965 vom Bundesamt für Statistik (BFS) die Ausdehnung der Erhebungen auf die wichtigsten Beherbergungsformen der Parahotellerie und erließ am 16. November 1978 die "Verordnung über die Fremdenverkehrsstatistik in der Parahotellerie», welche die zu erfassenden Beherbergungsformen eindeutig umschreibt. Aus finanziellen und personellen Gründen erfolgte der Aufbau der ParahotellerieStatistik nur schrittweise. Unvollständig ist die Erfassung heute nur noch im Teilbereich "Chalets und Ferienwohnungen", wogegen die Gäste in den übrigen Beherbergungsarten «vollständig» erfaßt werden.

Die schweizerische Statistik der Parahotellerie umfaßt heute:

\section{Chalets, Ferienwohnungen, Privatzimmer}

Die Datenerfassung erfolgt über Verkehrsvereine und Gemeinden und ist mit dem Einzug der Kurtaxen oder der Polizeimeldescheine gekoppelt. Die vollständige Erfassung der in Privatquartieren abgestiegenen Kundschaft erstreckt sich auf die Kantone Bern, Obwalden, Appenzell A.R., St.Gallen, Graubünden, Tessin, Wallis und Waadt sowie auf einige Fremdenverkehrsorte in anderen Kantonen.

\section{Campingplätze, Gruppenunterkünfte und}

Jugendherbergen (SJH)

Vorderhand werden gesamtschweizerische Ergebnisse der Parahotellerie nur jährlich publiziert. In dieser
Publikation sowie den alljährlich erscheinenden Berichten über jene Kantone, in welchen die Gäste aller Beherbergungsformen vollständig erfaßt werden, sind die Daten gegliedert nach Unterkunftsformen, Herkunftsländern, Winter- und Sommerhalbjahr und nach Fremdenverkehrsorten.

Die Arbeiten über die volkswirtschaftliche Bedeutung des Tourismus konzentrieren sich auf die Erstellung der Fremdenverkehrsbilanz, also auf die Ermittlung von Einnahmen und Ausgaben im Fremdenverkehr mit dem Ausland. Die Fremdenverkehrsbilanz umfaßt nach internationalen Konventionen folgende Hauptpositionen:

- Ferien- und Geschäftsaufenthalte;

- Kur-, Spital- und Studienaufenthalte;

- Tages- und Transitverkehr;

- internationale Personentransporte.

Vielfältig sind die übrigen Aufgaben der Sektion Tourismus. Sie reichen von den zahlreichen, vielfach mit arbeitsintensiven Sonderauszählungen und $\mathrm{Zu}$ sammenstellungen verbundenen Auskunftserteilungen über die Zusammenarbeit mit internationalen Gremien (OECD in Paris, Weltorganisation für Tourismus in Madrid usw.), Sonderaufgaben in Zusammenhang mit der Berechnung unseres Landesindexes der Konsumentenpreise bis zur Ermittlung der Frequenzen von ins Ausland gereisten Schweizern.

\section{Heinz Kleine}

Bundesamt für Statistik (BFS), Sektion Tourismus

Hallwylstr. 15, 3003 Bern, Tel. 031/61 8665

\title{
Die Schweizerische Verkehrszentrale (SVZ)
}

Vor dem Hintergrund der zunehmenden Bedeutung des Tourismus in unserem Land regte Nationalrat A.Seiler 1911 in einer Motion die Schaffung einer «Zentralstelle behufs Förderung des Fremdenverkehrs in der Schweiz» an. Mit der Gründung der «Nationalen Vereinigung zur Förderung des Reiseverkehrs» wurde der Vorschlag 1917 in die Tat umgesetzt. Ein Jahr später errichtete die neue, rein privatrechtlich strukturierte Organisation ihren Geschäftssitz unter der Bezeichnung «Schweizerische Verkehrszentrale (SVZ)" in Zürich. Wie schon zuvor betrieb man die Landeswerbung im Ausland über die Agenturen der Schweizerischen Bundesbahnen (SBB) in Paris, New York, Berlin, Kairo und Wien. Eine Ausnahme stellte lediglich Nizza dar, wo die SVZ 1923 ein eigenes Büro eröffnete. Als im Gefolge der Weltwirtschaftskrise auch die Logierfrequenzen in der Schweiz rapide sanken, drängte sich auf dem Gebiet der Auslandwerbung eine engere Zusammenarbeit der vorhandenen Verkehrsorganisationen auf.

1933 führten diese Bestrebungen zwischen den SBB, der SVZ und der PTT-Verwaltung zum Abschluß einer "Werbegemeinschaft», zu der sich wenig später auch noch das Eidgenössische Amt für Verkehr gesellte. Die SVZ war für die Bereitstellung des Propagandamaterials zuständig und erhielt das Recht, eigene Mitarbeiter in die SBB-Vertretungen zu entsenden, deren Zahl in den folgenden Jahren auf elf 
anstieg. Als wichtiges Datum in der Geschichte der SVZ ist der 21. September 1939 zu nennen, denn damals schuf das Parlament die Voraussetzungen, um das Agenturnetz der SBB der SVZ einzuverleiben, was 1941 erfolgte. Mit dem gleichen Bundesbeschluß wandelte man letztere in eine öffentlich-rechtliche Körperschaft um, die den Namen «Schweizerische Zentrale für Verkehrsförderung» erhielt. 1955 wurde schließlich wieder die ursprüngliche Bezeichnung «Schweizerische Verkehrszentrale» eingeführt, die bis heute verwendet wird.

Laut Organisationsstatut organisiert und führt die SVZ die gesamtschweizerische Verkehrswerbung im Inund Ausland durch, wobei auch das Verständnis für die politische, kulturelle und wirtschaftliche Eigenart der Schweiz geweckt werden soll. Zu diesem Zweck arbeitet die Verkehrszentrale auf lokaler, regionaler, kantonaler, nationaler und internationaler Ebene eng mit den verschiedensten Fachstellen und Kommissionen zusammen.

Getragen wird die SVZ in erster Linie vom Bund, der rund zwei Drittel an das Budget in der Höhe von 28,5 Mio. Franken (1983) beisteuert. Andere namhafte Träger sind die SBB, die PTT, der Schweizer HotelierVerein, die Schweizerische Straßenverkehrswirtschaft sowie die Swissair. $\mathrm{Zu}$ den übrigen der insgesamt etwas mehr als 400 Mitgliedern zählen alle 26 Kantone, eine Reihe von Gemeinden und vor allem die im Tourismus oder in dessen Umfeld tätigen Organisationen.
Am Hauptsitz in Zürich sind rund 50 Personen mit journalistischen Arbeiten, der Planung und Durchführung von Ausstellungen und touristischen Werbereisen, der Herstellung einer vielfältigen Palette an Informationsbroschüren, allgemeinen Publikationen, Fotos, Plakaten, Presseartikeln usw. sowie mit administrativen Aufgaben beschäftigt. Jährlich werden um die 600 Tonnen an Imprimaten an die Werbefront im Ausland, das heißt an die auf alle fünf Kontinente verteilten SVZ-Agenturen verschickt. In den insgesamt 24 Vertretungen sind 200 weitere Mitarbeiter tätig, denen insbesondere Informationsaufgaben sowie die Kontaktpflege mit den Medien und touristischen Vereinigungen des Gastlandes obliegen. Überdies ist ein Teil der Agenturen mit dem Verkauf von Billetten der Transportanstalten des öffentlichen Verkehrs beauftragt.

Die SVZ versucht nicht nur, mit ihren Partnern zusammen einen hohen und überzeugenden Wirkungsgrad der Tourismuswerbung zu erreichen, sondern sie ist auch bestrebt, in unserer Wirtschaftspolitik eine aktive Rolle zu spielen. Schließlich engagiert sich die Schweizerische Verkehrszentrale mit anderen Institutionen ganz generell für die Präsenz unseres Landes im Ausland, etwa nach dem Motto: «Die Schweiz - ein schönes Stück Europa».

Schweizerische Verkehrszentrale (SVZ)

Bellariastr. 38, 8027 Zürich, Tel. 01/202 3737

\section{Der Schweizerische Fremdenverkehrsverband (SFV)}

Der Schweizerische Fremdenverkehrsverband (SFV) wurde 1932 in einer für den Tourismus schwierigen Zeit gegründet. Als tourismuspolitischer Dachorganisation wurde ihm die Vertretung der mit dem Fremdenverkehr zusammenhängenden Gesamtinteressen übertragen. Oberste Zielsetzung des SFV ist somit, zu einer langfristigen Verbesserung des Angebotes des Reise- und Ferienlandes Schweiz und damit zur Förderung des drittwichtigsten Wirtschaftszweiges und des zweitwichtigsten Arbeitgebers beizutragen. Im Gegensatz zur Schweizerischen Verkehrszentrale oder zu den SBB verzichtet der SFV aber auf jegliche Publizität oder Tätigkeit, die der eigentlichen Reisewerbung und der Fremdenverkehrspropaganda dient. Der SFV ist als privatrechtlicher Verein organisiert, dem heute über 630 Mitglieder angehören. Er hat seinen Sitz in Bern und beschäftigt dort ein Team von zehn Mitarbeitern (Ökonomen, Juristen, PR-Fachleute und kaufmännisch ausgebildetes Personal).

Eine Hauptaufgabe des SFV besteht in der repräsentativen Vertretung der schweizerischen Fremdenverkehrswirtschaft. Dies setzt die Verbandszugehörigkeit möglichst vieler Tourismusträger voraus. Mit der Einsitznahme sämtlicher Kantone, von 75 Fremdenverkehrsgemeinden, über 200 nationalen, regionalen und lokalen touristischen Verbänden, Institutionen und Vereinen, 176 Bergbahnen, Hotels und Restaurants, 39 Banken und Versicherungen, 31 Architekturund Planungsbüros sowie von 78 sonstigen dem Fremdenverkehr nahestehenden Einzelunternehmungen in den Organen des SFV dürfte die Forderung nach einer umfassenden Mitgliedschaft erfüllt sein. 